\title{
Obtención y caracterización de capas de elevada dureza sobre fundiciones nodulares, mediante técnicas de difusión termorreactiva*
}

\section{Dayi Gilberto Agredo-Diaz ${ }^{1}$ \\ Arturo Barba-Pingarrón ${ }^{2}$ \\ Daniel Sánchez-Ruiz² \\ Jesús Rafael González-Parra ${ }^{2}$ \\ Raúl Gilberto Valdez-Navarro² \\ Jhon Jairo Olaya-Florez ${ }^{1}$ \\ Carlos Alberto González-Rodriguez ${ }^{3}$ \\ Alba Covelo-Villar ${ }^{2}$ \\ Miguel Ángel Hernández-Gallegos ${ }^{1}$ \\ Irma Angarita-Moncaleano ${ }^{1}$}

\author{
Universidad Nacional de Colombia \\ Sede Bogotá, Facultad de Ingeniería \\ Departamento de Ingeniería Mecánica y Mecatrónica. \\ Bogotá. \\ COLOMBIA \\ 2Universidad Nacional Autónoma de México \\ Facultad de Ingeniería, División de Ingeniería Mecánica e \\ Industrial, Centro de Ingeniería de Superficies y Acabados \\ (CENISA), Ciudad de México. \\ MÉXICO \\ ${ }^{3}$ Universidad Politécnica del Valle de México. \\ MÉXICO
}

correos electrónicos (emails):

dgagredod@unal.edu.co, arbapin5@gmail.com

gravemindflood117@gmail.com, rafael.parra@yandex.com

raulgvaldez@hotmail.com, jjolayaf@unal.edu.co

gorc74@yahoo.com, acovelov@gmail.com

mahg22@yahoo.com, iangaritam@unal.edu.co

Recibido 31-01-2020, aceptado 27-04-2020.

\section{Resumen}

En esta investigación se describen los trabajos llevados a cabo para generar capas, con presencia importante, principalmente, de carburo

*Este artículo es la versión extendida de los mejores trabajos del Congreso Nacional de Ingeniería Electromecánica y de Sistemas 2019 de niobio de elevada dureza, sobre una fundición nodular (FN) de matriz perlítica, mediante técnicas de difusión termorreactiva (TRD, por sus siglas en inglés). Los procesos se realizaron empleando un baño de bórax a 950 y $1025^{\circ} \mathrm{C}$, durante tiempos de 4 y 6 horas. Los sustratos se caracterizaron mediante espectroscopia de emisión atómica, microscopía óptica y microscopía electrónica de barrido. Las capas depositadas, asimismo, se caracterizaron empleando ensayos de microdureza Vickers, microscopía electrónica de barrido, espectrometría de dispersión de energía de rayos X (EDS por sus siglas en inglés) y difracción de rayos X (DRX). Se alcanzan valores de dureza de los recubrimientos del orden de 2600 Vickers con espesores cercanos a $25 \mu \mathrm{m}$. El análisis EDS muestra principalmente la presencia de $\mathrm{Fe}, \mathrm{Nb}, \mathrm{y} \mathrm{V}$. El proceso representa una alternativa de menor costo y ambientalmente más amigable que tecnologías como la de CVD y se considera una opción atractiva para dotar, entre otras características, de mayor resistencia al desgaste a la fundición nodular.

Palabras clave: carburo de niobio, difusión termorreactiva, DRX, fundición nodular, microscopía electrónica de barrido.

\section{Abstract}

(Obtaining and characterization of high hardness layers on Ductile Irons by means of Thermoreactive Diffusion techniques)

This research describes the work carried out to generate layers, with a significant presence of high hardness niobium carbide, on a pearlitic matrix ductile iron, by means of thermoreactive diffusion techniques (TRD). The processes were carried out using a borax bath at 950 and $1025^{\circ} \mathrm{C}$ for 4 and 6 hours. The substrates were characterized by atomic emission spectroscopy, optical microscopy, scanning electron microscopy. The deposited layers were characterized by vickers microhardness tests, scanning electron microscopy and X-ray energy dispersive spectrometry (EDS) and X-ray diffraction. The hardness values of coatings of the order of 2600 Vickers with thickness close to $25 \mu \mathrm{m}$, where the EDS analysis shows mainly the presence of $\mathrm{Fe}, \mathrm{Nb}$ and $\mathrm{V}$. The process represents a lower cost and more environmentally friendly alternative to technologies such as CVD and is considered an attractive option to provide greater wear resistance (and other characteristics), to ductile iron.

Index terms: ductile iron, niobium carbide, scanning electron microscopy, thermoreactive diffusion, XRD.

\section{Introducción}

Las fundiciones nodulares reúnen una serie de características que las hacen muy atractivas en un extenso campo de 
aplicaciones. El grafito libre que presentan, en forma de nódulos, otorga propiedades de alta conductividad térmica, características de autolubricación, capacidad para amortiguar de manera muy eficiente las vibraciones mecánicas, todo lo anterior unido a la posibilidad de modificar la matriz mediante tratamiento térmico, lo que permite satisfacer, cada vez más, diversos y exigentes requerimientos de la industria [1], [2]. Adicionalmente, las fundiciones nodulares poseen una densidad ligeramente menor que la de los aceros, pudiéndose fundir a temperaturas más bajas, lo cual conlleva una gran ventaja con respecto a materiales convencionales, reduciendo la energía necesaria para su procesamiento.

En años recientes, se ha conseguido mejorar aún más el desempeño de estos materiales, a través de la aplicación de un tratamiento térmico de austempering, por medio del cual se modifica su microestructura para generar una combinación de ausferrita y nódulos de grafito, lo que se conoce como fundición nodular austemperizada, (ADI, por sus siglas en inglés). Ello ha permitido mejorar, en mayor medida aún, sus propiedades mecánicas, logrando un muy buen balance de resistencia mecánica conjuntada con una buena tenacidad, lo que le ha abierto nuevas opciones de aplicación en varios campos. Incluso, las ADI se han venido utilizando en sustitución de algunos aceros aleados y de varias aleaciones de aluminio [3], [4] .

Por su parte, los tratamientos de difusión termorreactiva constituyen una variante, proveniente del campo de los tratamientos termoquímicos, por medio de la cual, es posible formar capas conteniendo, prioritariamente, carburos (y a veces carbonitruros), de elementos de alta afinidad por el carbono, tales como el vanadio o el niobio, compuestos que son de elevada dureza, con el propósito de colaborar a brindar una mayor microdureza, una elevada resistencia al desgaste y mejores propiedades superficiales [5], [6], [7].

A partir de lo anterior, en este trabajo se presentan los resultados obtenidos al generar capas, mediante la aplicación de técnicas de difusión termorreactiva, sobre una fundición nodular obteniendo capas, fundamentalmente, de $\mathrm{NbC}$. Las capas logradas fueron caracterizadas empleando técnicas de microscopía electrónica de barrido, análisis químico utilizando una microsonda de EDS, ensayos de microdureza y evaluación estructural usando difracción de rayos X, para la identificación de los compuestos y fases formadas.

\section{Metodología}

Para la obtención del recubrimiento se prepararon piezas de $2.5 \times 2.5 \mathrm{~cm}$ las cuales fueron sometidas a procesos de limpieza mediante etapas alcalinas y ácidas, más una limpieza ultra- sónica en acetona y fueron colocadas en un horno en el que previamente se encontraba bórax fundido, en un recipiente dispuesto para el tratamiento, a temperaturas de 950 y $1025^{\circ} \mathrm{C}$, durante tiempos de 4 y 6 horas, respectivamente, con contenido de ferroniobio y ferrovanadio $(8 \%$ en peso de cada una de las ferroaleaciones) en el baño y bórax, efectuándose un posterior enfriamiento al aire hasta temperatura ambiente.

La composición de la Fundición Nodular utilizada se obtuvo mediante espectroscopia de emisión atómica, en tanto la composición semicuantitativa del recubrimiento se determinó a través de EDS en un microscopio electrónico de barrido Philips XL20 utilizando una microsonda de EDS acoplada al microscopio. La preparación y caracterización metalográfica del sustrato se realizó siguiendo los lineamientos de la Norma ASTM E-3 [8], empleando etapas de lijado con papel de SiC desde la lija número 200 hasta la lija número 1000 , un posterior pulido con alúmina de 3 y $0.5 \mu \mathrm{m}$, y un ataque químico con nital al $2 \%$, seguido de la observación mediante microscopia óptica y microscopia electrónica de barrido (SEM). La caracterización morfológica de la capa se llevó a cabo mediante SEM, realizándose, asimismo, la medición del espesor de la capa promedio obtenida.

La caracterización estructural se realizó mediante difracción de rayos $\mathrm{X}$ en un difractómetro PanAlytical X'Pert Pro, operando a $45 \mathrm{kV}, 40 \mathrm{~mA}$, usando una radiación monocromática de $\mathrm{Cu}$, con una longitud de onda de 1,54 $\AA$, y un tamaño de paso de $0,02^{\circ}$ a un tiempo de $40 \mathrm{~s}$ por paso.

La dureza de las capas es obtenida mediante la aplicación del ensayo de microdureza bajo la norma ASTM E-384 [9] en un microdurómetro digital modelo HVS-1000, se usa una carga de $50 \mathrm{~g}$ y un tiempo de $10 \mathrm{~s}$.

\section{Resultados y discusión}

La composición elemental de la FN se muestra en la tabla 1, esta presenta un contenido de C de $3.606 \%$ y Si de $2.505 \%$, que representan valores típicos para un material de este tipo. La microestructura del material en estado de entrega (EE) permite evidenciar la distribución de fases presentes en el material, el cual está constituido por una matriz perlítica con la presencia de la fase ferrita y nódulos de grafito, estas mismas características son mostradas en los estudios consignados en [10], condición que se puede evidenciar en la figura 1. En la figura 2 se muestra, en una imagen de microscopía electrónica de barrido, una magnificación que permite evidenciar de forma más clara la presencia mayoritaria de la perlita y como la ferrita aparece rodeando a los nódulos de grafito en lo que se conoce como "morfología ojo de buey". 
Tabla 1. Composición elemental del material de estudio. Porcentaje en peso.

\begin{tabular}{ccccc}
\hline \%Fe & \% C & \% Si & \% Mn & \% P \\
\hline 92.7 & 3.606 & 2.505 & 0.521 & 0.017 \\
\hline
\end{tabular}

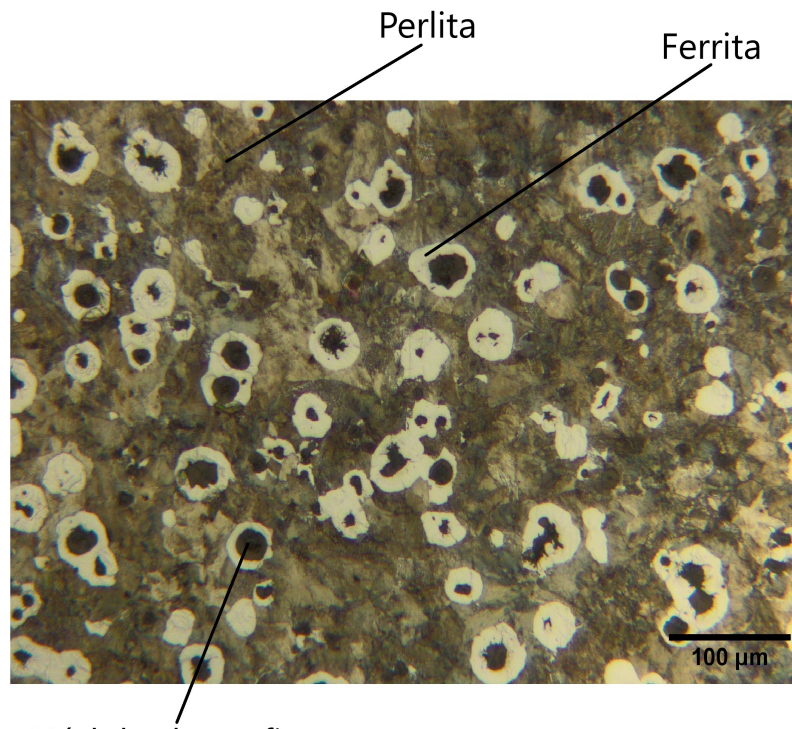

Nódulo de grafito

Fig. 1. Microestructura de la fundición nodular en estado de entrega mediante microscopia óptica.

La figura 3 muestra la obtención de una monocapa que crece de forma perpendicular a la superficie de la fundición, se evidencia un recubrimiento homogéneo, compacto y con buena

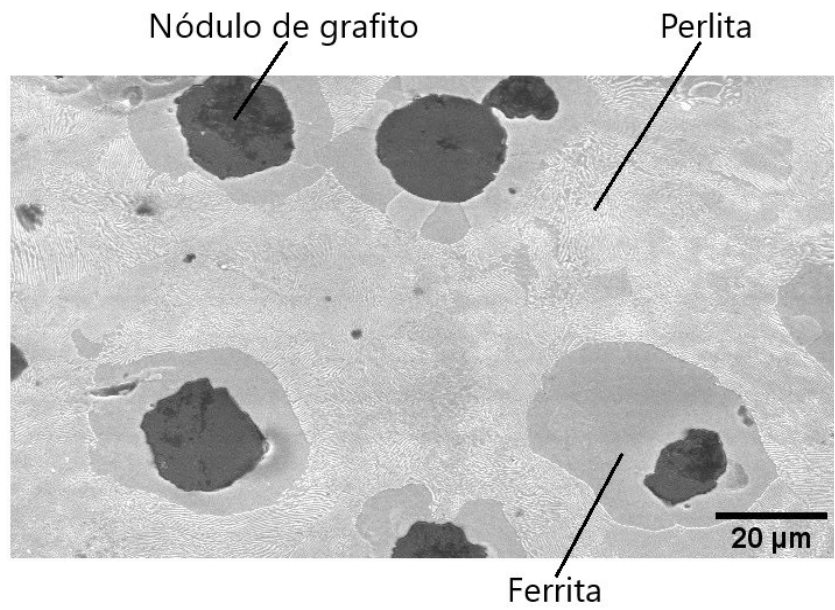

Fig. 2. Microestructura del material en estado de entrega mediante SEM con electrones secundarios (SE).

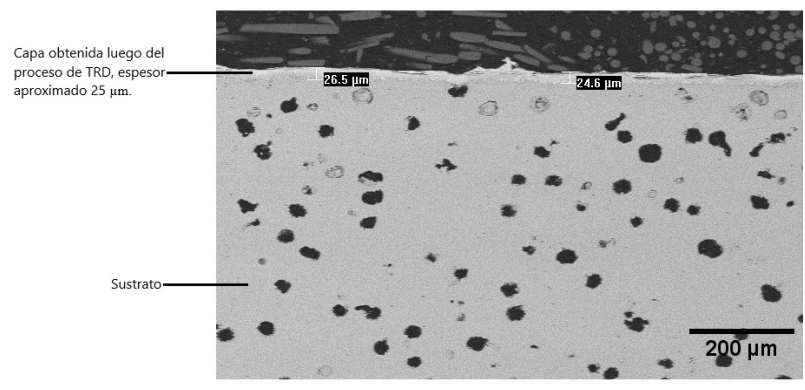

Fig. 3. Imagen de microscopía electrónica de barrido de la capa obtenida mediante difusión termorreactiva, sobre una fundición nodular (SEM, electrones retrodispersados (BSE)). La capa es homogénea en espesor.

adherencia al sustrato [6], donde el crecimiento de la capa está controlado por la difusión de átomos de carbono en la capa del carburo que se está formando y, a su vez, por la difusión de los átomos del metal en la capa de carburo, estas condiciones hacen que se propicie la formación de una capa de alta adherencia [11]. Se destaca la obtención de un espesor del orden de $25 \pm 1 \mu \mathrm{m}$, lo cual es congruente con lo obtenido en [12], [13], [14], [21].

La figura 4 muestra el microanálisis de EDS para la capa, se evidencia la presencia mayoritaria de hierro, niobio y vanadio producto de las ferroaleaciones, la aparición de $\mathrm{C}$ es un indicador inicial de la posible formación de carburos, lo cual es proveniente de la elevada afinidad del $\mathrm{Nb}$ y el $\mathrm{V}$ por el C, a su vez la presencia de boro proviene del baño de bórax usado en el tratamiento. Estos resultados son comparables a los obtenidos en las investigaciones realizadas por Castillejo y colaboradores [15].

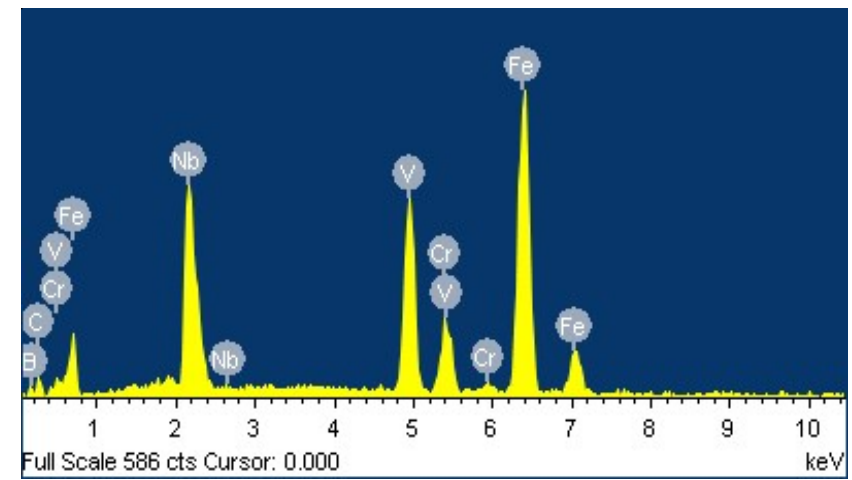

Fig. 4. Espectro de EDS obtenido para la capa generada sobre FN. Destacan la presencia de $\mathrm{Nb}$ y $\mathrm{V}$, así como $\mathrm{Fe}, \mathrm{B}$ y $\mathrm{Cr}$. 


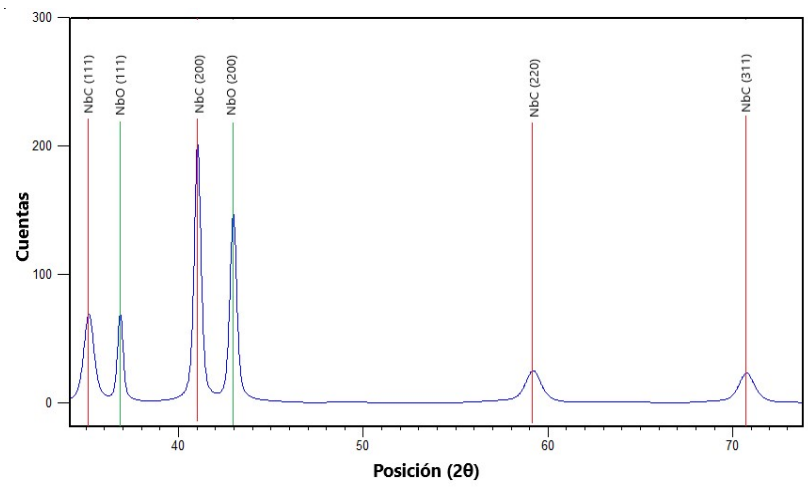

Fig. 5. Patrón de difracción de rayos $X$ para la capa obtenida sobre una fundición nodular, mediante difusión termorreactiva. Se observa claramente la presencia del carburo de niobio y de óxido de niobio en la muestra tratada.

La figura 5 muestra el patrón de difracción de las capas logradas. Se verifica la presencia de $\mathrm{NbC}$ según el patrón de referencia ICDD 01-074-1222 y NbO según el patrón de referencia ICDD 01078-0642, ambos con estructura cristalina cúbica. La posición angular $(2 \theta)$, con su correspondiente plano se muestran en la tabla 2. Las mayores intensidades corresponden a las orientaciones de los planos (200) y (111), y una presencia menor de las orientaciones (220) y (311) del NbC. La formación de óxido de niobio $(\mathrm{NbO})$ está ligado a la reacción que ocurre a las elevadas temperaturas del proceso, estudios confirman que a temperaturas cercanas a los $1000^{\circ} \mathrm{C}$, el elemento formador de carburo se oxida rápidamente lo que propicia la formación de óxidos del metal de las ferroaleaciones, esto se reporta en [11] y de manera particular en [16]y [17].

La formación de estas capas con la estructura cristalina cúbica mencionada, asimismo, se atribuye a la elevada afinidad del niobio por el carbono, como se reporta también en [16], [17], en conjunto con un adecuado control de los parámetros termodi-

Tabla 2. Posición de los picos para el NbC y NbO.

\begin{tabular}{cccc}
\hline \multirow{2}{*}{ Compuesto } & Plano (hkl) & \multicolumn{2}{c}{ Angulo 28 } \\
& & Teórico & Experimental \\
\hline \multirow{3}{*}{$\mathrm{NbC}$} & 111 & 35.3 & 35.12 \\
& 200 & 40.99 & 40.99 \\
& 220 & 59.36 & 59.14 \\
& 311 & 70.99 & 70.68 \\
\hline \multirow{2}{*}{$\mathrm{NbO}$} & 111 & 36.95 & 36.82 \\
& 311 & 42.93 & 42.93 \\
\hline
\end{tabular}

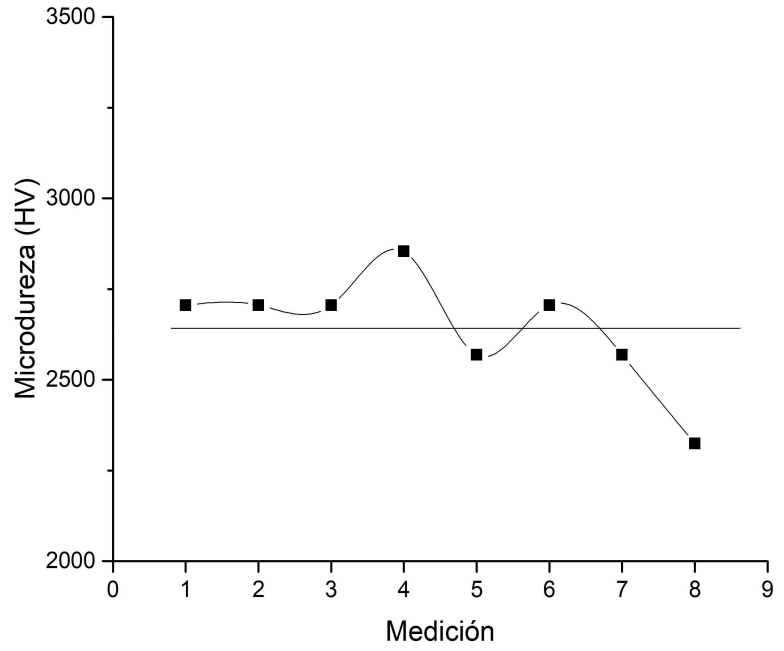

Fig. 6. Microdureza de la capa obtenida mediante TRD sobre la fundición nodulara.

námicos que intervienen en el proceso de crecimiento de la capa [4], [6] y [15].

La figura 6 presenta el resultado producido a través de la aplicación del ensayo de microdureza sobre diversas zonas de las capas obtenidas, y los resultados reportados (del orden de 2600 Vickers) estarían en concordancia con la formación de Carburo de Niobio y con resultados reportados en las referencias [15], [12], [18], [19], [16], [17], [20]. En un siguiente trabajo se evaluará el efecto de estos tratamientos, en primera instancia, en la resistencia al desgaste, la cual se espera pueda mejorar con la presencia de estas capas.

\section{Conclusiones}

Ha sido posible obtener capas de elevada dureza, sobre una fundición nodular, mediante tecnologías de difusión termorreactiva, empleando un baño de bórax, con adición de pequeñas cantidades de ferroaleaciones, conteniendo elementos que son fuertes formadores de carburos, tales como niobio o vanadio, siendo esta una buena alternativa para la protección de componentes sometidos a condiciones de desgaste.

Se obtiene una monocapa del orden de 25 micrómetros de espesor, con crecimiento normal a la superficie del sustrato, de buena homogeneidad, compacta y con buena adherencia. El microanálisis de EDS muestra la presencia de cantidades significativas de hierro, niobio, y vanadio en la capa, obteniendo valores de microdureza del orden de los $2600 \mathrm{HV}$, lo cual va ligado a la formación de $\mathrm{NbC}$, esto se confirmó mediante difracción de rayos $\mathrm{X}$. 
La presencia $\mathrm{NbO}$ en menor cantidad está dada por el elemento formador del carburo que se oxida rápidamente, este se une al $\mathrm{Nb}$ contenido en la ferroaleación y propicia la formación del óxido.

Un siguiente trabajo incluirá la evaluación de la resistencia al desgaste y a la corrosión, en diversos medios, de las capas logradas.

\section{Agradecimientos}

Los autores del Centro de Ingeniería de Superficies y Acabados (CENISA) de la Facultad de Ingeniería de la UNAM, agradecen el apoyo otorgado para la realización de esta investigación, por parte de la Dirección General de Asuntos del Personal Académico de la UNAM, a través de los Proyectos PAPIIT IT101318 "Desarrollo de Tecnologías de Superficie para la Optimización de Componentes y Sistemas" y PAPIME PE100218 "Programa para la Actualización y Mejora de la Enseñanza y Aprendizaje de Temas de Materiales, Manufactura e Ingeniería de Superficies a Estudiantes de Ingeniería".

\section{Referencias}

[1] R. Castillo, V. Bermont, V. Martínez, "Relaciones entre la microestructura y las propiedades mecánicas en fundiciones nodulares: Revision bibliográfica," Rev. Metal., vol. 35, núm. 5, pp. 329-334, 1999.

[2] D. G. Agredo Diaz, A. Barba Pingarrón, J. R. Gonzalez Parra, J. J. Olaya Florez, R. G. Valdez Navarro, "Evaluación de la resistencia a la corrosión de una fundición nodular recubierta por proyección térmica," Memoria del congreso Internacional de Ingeniería Mecánica y Mecatrónica, (CIMM/CIIMA) 2019, p. 121.

[3] T. Arai, "The thermo-reactive deposition and diffusion process for coating steels to improve wear resistance. Capítulo 19", en Thermochemical Surface Engineering of Steels: Woodhead Publishing Limited, 2015, pp. 703-736.

[4] J. R. Davis, "Thermal Diffusion Process," Encycl. Tribol., pp. 3586-3586, 2013.

[5] F. Castillejo, D. Marulanda, J. Olaya, "Estudio de recubrimientos de carburos ternarios de niobio-vanadio producidos sobre acero D2 usando la técnica de deposición por difusión termorreactiva," Rev. Latinoam. Metal. y Mater, vol. 34, núm. 2, pp. 230-239, 2014.

[6] F. E. Castillejo Nieto, J. J. Olaya Flórez, "Recubrimientos de VC y NBC producidos por DRT: Tecnología económica, eficiente y ambientalmente limpia," Cienc. e Ing. Neogranadina, vol. 22, núm. 1, p. 95, 2012.

[7] M. Aghaie-Khafri and F. Fazlalipour, "Vanadium carbide coatings on die steel deposited by the thermo-reactive diffusion technique," J. Phys. Chem. Solids, vol. 69, núm. 10, pp. 2465-2470, 2008.
[8] ASTM, "E3 Preparation of Metallographic Specimens," Annu. B. ASTM Stand., vol. 11, núm. Reapproved 2017, pp. 1-17, 2017.

[9] ASTM, Standard Test Method for Microindentation Hardness of Materials. 2008, pp. 281-293.

[10] C. Labrecque, M. Gagné, "Ductile Iron: Fifty years of continuous development," Can. Metall. Q., vol. 37, núm. 5, pp. 343-378, 1998.

[11] F. E. Castillejo Nieto, Recubrimientos de Carburos Ternarios Depositados con la Técnica TRD Rec, Universidad Nacional de Colombia, 2013.

[12] F. A. P. Fernandes, J. Gallego, C. A. Picon, G. Tremiliosi Filho, L. C. Casteletti, "Wear and corrosion of niobium carbide coated AISI 52100 bearing steel," Surf. Coatings Technol., vol. 279, pp. 112-117, 2015.

[13] X. Liu, H. Wang, D. Li, Y. Wu, "Study on kinetics of carbide coating growth by thermal diffusion process," Surf. Coatings Technol., vol. 201, núm. 6, pp. 2414-2418, 2006.

[14] C. Y. Wei, F. S. Chen, "Thermoreactive deposition/ diffusion coating of chromium carbide by contact-free method," Mater. Chem. Phys., vol. 91, núm. 1, pp. 192199, 2005.

[15] F. E. Castillejo-Nieto, J. J. Olaya-Florez, J. E. Alfonso, "Wear resistance of vanadium-niobium carbide layers grown via TRD," Dyna, vol. 82, núm. 193, pp. 104-109, 2015.

[16] A. Bedolla-Jacuinde, "Niobium in Cast Irons," en Progress in Metallic Alloys in IntechOpen, 2016, pp. 187-219.

[17] C. Soares, F. E. Mariani, L. C. Casteletti, A. N. Lombardi, G. E. Totten, "Characterization of Niobium Carbide Layers Produced in Ductile Cast Iron Using ThermoReactive Treatments," Mater. Perform. Charact., vol. 6, núm. 4, p. 20160093, 2017.

[18] M. Ariati, W. N. Putra, A. Aryowiweko, "Effect of FeCr/ FeV Ratio During Thermo-Reactive Deposition(TRD) Process on Mechanical Properties and Characteristics of Carbide Layer of SUJ2 Steel," Mater. Today Proc., vol. 17, pp. 1736-1742, 2019.

[19] R. M. Triani, F. E. Mariani, L. F. De Assis Gomes, P. G. B. De Oliveira, G. E. Totten, L. C. Casteletti, "Improvement of the tribological characteristics of AISI 8620, 8640 and 52100 steels through thermo-reactive treatments," Lubricants, vol. 7, núm. 8, 2019.

[20] O. Susarrey, A. López, L. Morales, D. Samayoa, J. Martínez, G. Montes de Oca, O. Flores, I. Campos, J. Silva, A. Balankin, "Tópicos selectos de mecánica de la fractura," Cientifica, vol. 7, núm. 3, julio-septiembre 2003. pp. 181-200.

[21] I. Campos, R. Torres, G. Ramírez, L. Zuñiga, M. E. Sánchez, "Evaluación de la movilidad del boro en aceros al carbono y herramienta en el proceso de borurización en pasta," Científica, vol. 10, núm. 3, 2006. 
